\title{
Active Uncertainty Reduction during Gripping using Range Cameras- Dual Control
}

\author{
Bernt Nilsson ${ }^{1}$, Åke Wernersson ${ }^{2,1}$ \\ ${ }^{\mathrm{I}}$ Robotics and Autonomous Mechanical Systems \\ IKP, Linköping University, 58183 Linköping Sweden \\ email:berni@ikp.liu.se fax:int 46-13281101 \\ ${ }^{2}$ Robotics \& Automation, Luleå University of Technology \\ S-97187 Luleå, Sweden, email:robotics@sm.luth.se
}

\begin{abstract}
This paper is on sensor based control for guiding a robot to correct gripping of objects having a large position uncertainty. An eye-in-hand mounted range camera is considered. A probabilistic problem formulation based on the requested posture at gripping and corresponding tolerances is presented. The problem is solved approximately using dynamic programming for a 1-degree-of-freedom manipulator. A five-step dual control law is studied in more detail. A typical case is that in the first part of the control sequence the robot steers towards the optimal sensing position and in the last part the error with respect to the gripping posture is minimized. Since range camera sensing introduces both range dependent noise and occlusion there is a need for 'exploratory moves'. This behavior is formalized and includes 'dual control'.
\end{abstract}

Keywords: gripping tolerances, range camera, range dependent noise, exploratory moves, dual control, probing

\section{INTRODUCTION-THE PROBLEM}

Typical gripping operations for a robot can be made more robust by a visual sensor guided control system [6],[10]. Small and smart sensors for 'in hand use' are now becoming available. However new problems also arise, i.e. spuriouses and ambiguities in sensor readings [5] and lack of complete information for decision making [2]. In this paper we will focus on the latter and derive a feedback controller for positioning of a robot relative to an object with a large position uncertainty, e.g. Figure 1. Much like [12] the basic idea is to design a task driven feedback controller. Not necessarily with complete object recognition and world modelling like [11]. The sensor considered is an eyein-hand mounted range camera. The positioning problem is specified by the requested posture at gripping and the corresponding tolerances. For the 1-degree-of-freedom case an approximative solution is derived using dynamic programming. The solution is evaluated in detail for five control steps. Typically, the robot first minimizes the uncertainty by positioning the robot at the optimal sensing position and in the last part the robot moves to the gripping point.

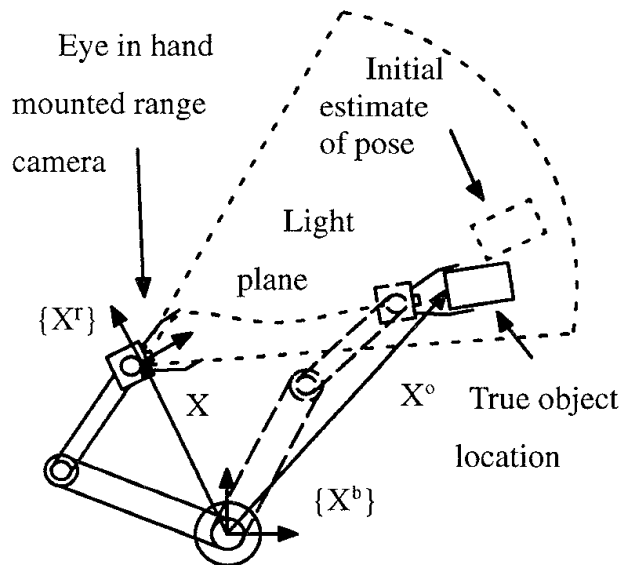

Figure 1. A gripping task with large initial uncertainty illustrated in the $(x, y, \Theta)$-space for a 3-DOF manipulator. The relative position between the object and the gripper is to be reduced by sensor feedback from a range camera. Range dependent noise and occlusion introduces 'exploratory moves'.

\section{PROBLEM FORMULATION}

The sensor is a range camera mounted in the gripper. From the large amount of range data $z_{k}$ obtained at time $t_{k}$ the relative posture $X_{o}-X_{k}$ of the object is extracted by triangulation giving

$$
z_{k}=h\left(X_{0}-X_{k}\right)+v_{k}
$$

where

$$
\begin{aligned}
& X_{k}=\text { robot posture at time } t_{k} . \\
& X_{o}=\text { object posture. } \\
& v_{k}=\text { measurement error in extraction. }
\end{aligned}
$$

The function $h$ is non-linear and will be derived in section 3. To describe the motion of the robot a kinematical state model

$$
x_{k+1}=f\left(x_{k}, u_{k}, w_{k}\right)
$$

is used where

$x_{k}=X_{o}-X_{k}$, i.e. the relative posture at time $t_{k}$.

$u_{k}=$ velocity control signal at time $t_{k}$.

$w_{k}=$ motion error at time $t_{k}$. 
The motion model $f$ will be derived in section 4 . Given (1) and (2) the gripping problem is formulated as

Problem 1. Find an admissable control strategy $\left(\mathrm{u}_{0}, \ldots, \mathrm{u}_{\mathrm{N}-1}\right)$ for the system given by (1) and (2) using the observed sequence $\left(z_{0}, \ldots, z_{N-1}\right)$ and $p\left(x_{0}\right)$ such that $\operatorname{Prob}\left(\left(x_{N}-x_{g}\right) \notin A\right)<p_{f}$

where

$x_{N}=X_{o}-X_{N}$, i.e. relative posture at time $t_{N}$.

$x_{g}=$ requested relative posture at gripping.

$A=$ compact set specified by the tolerances $\epsilon$.

$\epsilon=\left[\epsilon_{1}, \ldots, \epsilon_{n}\right]^{T}$ tolerances requested at gripping.

$p\left(x_{0}\right)=$ probability density function, (p.d.f.) of initial

relative posture.

$p_{f}=$ probability of failure, close to zero.

$t_{N}=$ final time when the robot grips the object.

Note that $x_{N}$ and $x_{g}$ are $n \times 1$ vectors given with respect to a frame $\left\{X^{r}\right\}$ in the robot gripper. The tolerances $\epsilon$ is an $n \times 1$ vector of required final accuracy for each member of $x_{N}$. The set $A$ is typically rectangular, i.e. a box for $n=3$.

Problem 1, as formulated, is complicated and 'non-standard'. Thus we approach problem 1 by solving the following minimization problem.

Problem 2. Find an admissable control strategy $\left(\mathrm{u}_{0}, \ldots, \mathrm{u}_{\mathrm{N}-1}\right)$ for the system given by (1) and (2) using the observed sequence $\left(z_{0}, \ldots, z_{N-1}\right)$ and $p\left(x_{0}\right)$ such that the quadratic cost functional (3) is minimized.

$$
\begin{aligned}
& J\left(u_{0}, u_{1}, . ., u_{N-1}\right)=E\left[\left(x_{N}-x_{g}\right)^{T} S_{N}\left(x_{N}-x_{g}\right)+\right. \\
& \left.\sum_{k=0}^{N-1}\left(\left(x_{k}-x_{r}\right)^{T} S_{k}\left(x_{k}-x_{r}\right)+u_{k}^{T} M_{k} u_{k}\right)\right]
\end{aligned}
$$

where $x_{r}$ denotes a reference trajectory and $S_{k}$ and $M_{k}$ are tuning parameters. Further, an admissable control strategy must be defined.

Admissable control candidates at time $t_{k}$ are all control signals $u_{k}$ which are functions of observed outputs and applied control signals up to time $t_{k-1}$, i.e. of $I_{k-1}=\left[z_{0}, \ldots, z_{k-1}, u_{0}, \ldots, u_{k-1}\right]$, and of the a priori data.

In this paper we will solve problem 2 for a 1-degreeof-freedom robot, i.e. $n=1$. Normally the minimization of (3) is constrained, i.e. $u \in U$. However if the object and the requested gripping posture is well inside the robots work space, the minimization problem can be solved unconstrained.

\section{THE RANGE CAMERA MODEL}

The range sensor consists of a laser with a cylindrical lens to generate a light plane and a CCD-camera for sensing. The basic principle is that the diffuse reflection from objects in the light plane are detected by the camera. From the known geometry of the setup of camera and laser and the image coordinates the location of the objects can easily be found by triangulation. Below we will only examine the sensor along the $X^{r}$ axis in the light plane, Figure 2. Note

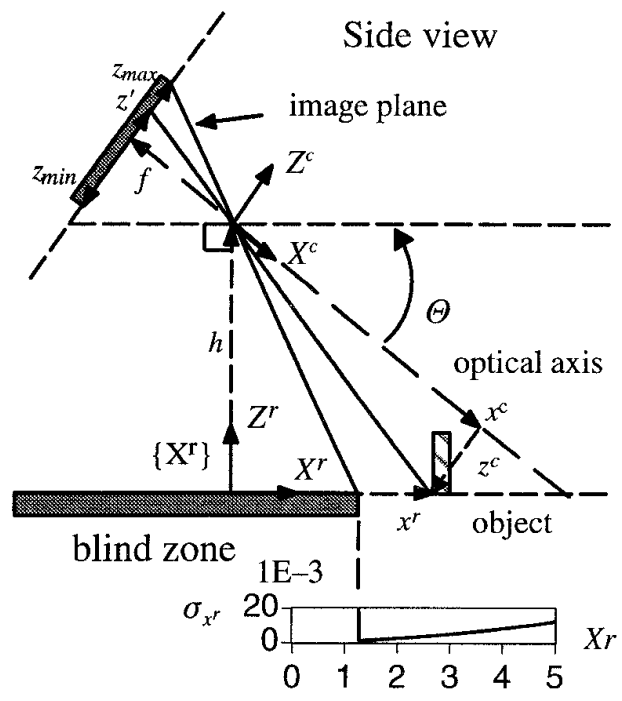

Figure 2. The range camera principle, based on the pin hole camera model. The distance $x^{r}$ is given by triangulation. Below, the standard deviation of the camera noise is propagated to the $X^{r}$-axis. The noise increase as $x^{2}$ with respect to distance, also the step increase for dropouts inside the blind zone is illustrated.

also that there is a blind zone close to the sensor.

The range camera parameters are

$h=$ orthogonal distance from the camera to the light plane.

$\Theta=$ angle between the light plane and the optical axis. $f=$ focal distance, assumed known from calibration.

From the geometry in Figure 2, and since objects are constrained to $z^{r}=0$ the following projection equation is derived.

$$
z^{\prime}=f \frac{x^{r} \sin \Theta-h \cos \Theta}{h \sin \Theta+x^{r} \cos \Theta}
$$

where $x^{r}$ is the distance to the object in the coordinates of the robot gripper. The projection (4) is valid for all values of $x^{r}$ but is singular at $x^{r}=-h \tan \Theta$. However for a real range camera the image plane is limited, i.e. only $z^{\prime} \in\left[z_{\min }, z_{\max }\right]$ is possible to receive. This bounds the cameras field of view both for small $x^{r}$ and large $x^{r}$, Figure 2.

Eq. (4) only gives the noise free description of the sensor signal $z^{\prime}$, some error sources are

1. The quantization in image coordinates.

2. Inaccurate calibration, i.e. $h, \Theta$ or $f$ are not known exactly

3. Lens distortion 
Neglecting the last two error sources and further reduce the amount of parameters by the following change of variables.

$$
z=\frac{z^{\prime}}{f} \quad x=\frac{x^{r}}{h}
$$

The change of variables also has the benefit of making both $z$ and $x$ non-dimensional.

Since the output is the image coordinate $z^{\prime}$, scaled by $f$, it is reasonable to assume that the measurement error is independent of range $x$. Further $z^{\prime}$ is extracted by peek detection. No sub pixel algorithm is currently used. Also assume the laser detection to be within one pixel width. Consequently the measurement noise can be modelled as uniform within the detected pixel, hence

$$
p\left(v_{k}\right)=U\left(0, R_{k}\right)=U\left(0, \frac{1}{f^{2} 12}\right)
$$

Here $U$ is short for uniform and $R_{k}$ denotes the variance. This gives the following measurement equations

$$
z_{k}=\frac{x_{k} \sin \Theta-\cos \Theta}{x_{k} \cos \Theta+\sin \Theta}+v_{k}=h\left(x_{k}\right)+v_{k}
$$

Note that so far we have not modelled the range cameras limited field of view. Returning to the original variables $z^{\prime}$ and $x^{r}$ for a moment. In [4] and [8] the measurement noise in $z$ is propagated to $x^{r}$ giving

$$
\sigma_{x^{r}} \approx \frac{\left(h \sin \Theta+\hat{x}^{r} \cos \Theta\right)^{2}}{h f} \sigma_{z^{\prime}} \approx \frac{\left(\hat{x}^{r} \cos \Theta\right)^{2}}{h f} \sigma_{z^{\prime}}
$$

where $\sigma_{z}$ is the standard deviation of error in $z^{\prime}$ and $\hat{x}^{r}$ is the mean of $x^{r}$. The last approximation is only valid for large $x^{r}$. Clearly the error in range $x^{r}$ is proportional to the range squared and inversely proportional to the baseline $h$ and the focal distance $f$, Figure 2 . As pointed out in [8] in order to decrease $\sigma_{x^{r}}, h$ and $f$ can not always be made as large as wanted. $f$ limits the field of view and $h$ is limited by mechanical properties and also enhance the risk for occlusion. From (8) it is also evident that the optimal sensing position must be just in front of the blind zone at $x_{\min }$.

\section{ROBOT MOTION MODEL}

In order to keep the first results transparent and the complexity low a 1-degree-of-freedom robot is considered.

The robots position $x$ is modeled as the first order 'stochastic' differential equation

$$
\frac{d x}{d t}=u(t)+b u(t) \dot{w}_{1}(t)+c \dot{w}_{2}(t)
$$

where $x$ is the position of the object in the coordinates of the moving robot and $u$ is the velocity control signal. The last two terms in (9) are stochastic, included to model motion uncertainty. The sequences $\left\{\dot{w}_{1}(t)\right\}$ and $\left\{w_{2}(t)\right\}$ are continuous white processes and $b$ and $c$ are modeling parameters. Assuming that the control signal $u(t)$ is constant during the sampling interval, (9) gives the following linear discrete time model.

$$
\begin{aligned}
& x_{k+1}=x_{k}+\int_{t_{k}}^{t_{k+1}} u(s)+u(s) b \dot{w}_{1}(s)+c \dot{w}_{2}(s) d s= \\
& x_{k}+T u\left(t_{k}\right)+u\left(t_{k}\right) b\left(w_{1}\left(t_{k+1}\right)-w_{1}\left(t_{k+1}\right)\right)+ \\
& c\left(w_{2}\left(t_{k+1}\right)-w_{2}\left(t_{k+1}\right)\right)= \\
& x_{k}+T u_{k}+b u_{k} \sqrt{T} \tilde{w} 1_{k}+c \sqrt{T} \tilde{w} 2_{k}
\end{aligned}
$$

The last equality follows from the properties of the Wiener process, $w(t)$. Further $\left\{\tilde{w} 1_{k}\right\}$ and $\left\{\tilde{w} 2_{k}\right\}$ are sequences of independent normal random variables with zero mean and covariances

$$
E\left[\tilde{w} 1_{k}^{2}\right]=1 \quad E\left[\tilde{w} 2_{k}^{2}\right]=1 \quad E\left[\tilde{w} 1_{k} \tilde{w} 2_{k}\right]=0
$$

With uniform sampling (10) adds one part proportional to the control signal $u_{k}$ and one constant part to each step. The first part can be used to model increasing uncertainty for high velocities. The last part which adds a constant uncertainty $c^{2} T$ for each sample can be used to model play in the robot links. An example of a similar noise model, for a mobile robot, is given in [4].

\section{STATE ESTIMATION}

It is at all time assumed that the feedback controller can be separated into a state estimator in sequence with a control law, Figure 3 . Here $I_{k-1}=\left[z_{0}, \ldots, z_{k-1}, u_{0}, \ldots, u_{k-1}\right]$ $k=1,2, \ldots N-1$, i.e. denotes the available information for control at time $t_{k}$.

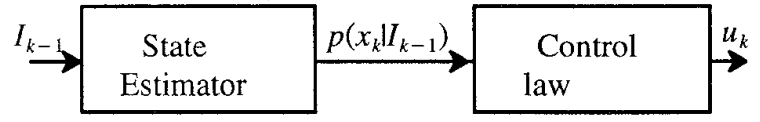

Figure 3. The structure of the feedback controller. The information available for control at time $t_{k}$ is used to update the probability density function of $x_{k}$. Subsequently the control law is based on $p\left(x_{k} \mid I_{k-1}\right)$.

The purpose of the state estimator is to calculate the p.d.f. $p\left(x_{k} \mid I_{k-1}\right)$ containing all information needed for calculating the control law. There are however two complications, the measurement equation (7) is non-linear and $z_{k}$ only exist within $\left[z_{\min }, z_{\max }\right]$. First introduce $\gamma_{k}$ to indicate when a measurement $z_{k}$ is defined, hence

$$
\gamma\left(z_{k}\right)=\left[\begin{array}{ll}
1 & \left(z_{\min } \leq z_{k} \leq z_{\max }\right) \\
0 & \left(z_{k}<z_{\min }\right) \text { or }\left(z_{k}>z_{\max }\right)
\end{array}\right.
$$

At time $t_{k}$, conditioned that $\gamma_{k}=1$, the measurement equation (7) can be linearized and used in a Kalman filter to calculate an approximation of $p\left(x_{k} \mid I_{k-1}\right)$. Define $\hat{x}_{k \mid k-1}$ as the position estimate of the object relative to the robot at time $t_{k}$. Then (7) is linearized by expanding it in a Taylor series about $\hat{x}_{k \mid k-1}$. The first two terms gives 


$$
\begin{aligned}
& z_{k} \approx h\left(\hat{x}_{k \mid k-1}\right)+H_{k}\left(x_{k}-\hat{x}_{k \mid k-1}\right)+v_{k} \\
& \quad \text { where } \\
& H_{k}=\left.\frac{\partial h}{\partial x_{k}}\right|_{x_{k}=\hat{x}_{k \mid k-1}}=\frac{1}{\left(\hat{x}_{k \mid k-1} \cos \Theta+\sin \Theta\right)^{2}}
\end{aligned}
$$

If now the higher order terms in (7) are small around the mean of $x_{k}$, compared to the variance of $x_{k},(13)$ can be used as a linear approximation. This is most restrictive for high inital variance at close range since there $h(x)$ can be very non-linear depending on camera parameters. Further assume that the measurement noise in (13) and the processs noise in (11) are modeled as white noise processes. Assume also that the unknown initial position $x_{0}$ is normal, i.e. $N\left(\hat{x}_{0}, \Sigma_{0}\right)$. The signal model (11) and (13) then fits the Kalman filter framework. The algorithm for updating the Kalman filter is given by [3], hence

$$
\begin{aligned}
& \hat{x}_{k+11 k}=\hat{x}_{k \mid k-1}+u_{k} T+ \\
& \frac{\sum_{k \mid k-1} H_{k}}{H_{k}^{2} \Sigma_{k \mid k-1}+R_{k}}\left(z_{k}-h\left(\hat{x}_{k \mid k-1}\right)\right) \gamma\left(z_{k}\right) \\
& \sum_{k+1 \mid k}=\Sigma_{k \mid k-1}\left(1-\gamma\left(z_{k}\right)\right)+ \\
& \frac{R_{k} \Sigma_{k \mid k-1}}{H_{k}^{2} \sum_{k \mid k-1}+R_{k}} \gamma\left(z_{k}\right)+b^{2} u_{k}^{2} T+c^{2} T
\end{aligned}
$$

where $\gamma\left(z_{k}\right)$ is given by (12).

In contrast with a signal model that is truly linear the output of the filter i.e., the position estimate $\hat{x}_{k+1 k k}$ and corresponding variance $\Sigma_{k+1 k k}$, are approximations, hence

$$
\begin{aligned}
& \hat{x}_{k+1 \mid k} \approx E\left[x_{k+1} \mid I_{k}\right] \\
& \Sigma_{k+11 k} \approx E\left\{\left[x_{k+1}-\hat{x}_{k+11 k}\right]^{2} \mid I_{k}\right\} \\
& \quad \text { also } \\
& p\left(x_{k+1} I_{k}\right) \approx N\left(\hat{x}_{k+1 \mid k}, \Sigma_{k+11 k}\right)
\end{aligned}
$$

Remark: In the estimator above we have modelled $z_{k}$ as a normal distributed random variable, after linearization, and not limited by the field of view $\left[z_{\min }, z_{\max }\right]$. Instead we have used $\gamma\left(z_{k}\right)$ to describe when $z_{k}$ is available, i.e. inside the field of view $\left[z_{\min }, z_{\max }\right]$. This is an approximation, the true probability density function of $z_{k}$ must be zero outside $\left[z_{\min }, z_{\max }\right]$. One could argue that if a measurement $z_{k}$ is missing it implies that $\gamma\left(z_{k}\right)=0$ and this fact includes information about the true position $x_{k}$. However since there are many other unmodelled properties for a missing $z_{k}$, i.e. changing object surface properties, malfunctions etc., this information is excluded.

Since $H_{k}$ in (14) includes $\hat{x}_{k \mid k-1}$ which depends on $u_{k-1}$ it is clear that the value of the position variance $\Sigma_{k+1 \mid k}$ will depend on $u_{k-1}$. This property arise since the measurement model is non-linear. In [1] it is shown that for an exact linear system with quadratic cost the accuracy of the state estimate can not be affected by the control signal, i.e. the 'certainty equivalence' principle. When designing the control law this distinction from a linear system will be taken into account. Note also that in (16), outside the cameras field of view, $\Sigma_{k+1 \mid k}=\Sigma_{k \mid k-1}+b^{2} u_{k}^{2} T+c^{2} T$ and consequently no reduction of uncertainty is possible.

\section{THE CONTROL LAW}

The second part of the feedback controller will now be derived, i.e. the mapping from $p\left(x_{k} \mid I_{k-1}\right)$ to the control $u_{k}$. In order to reduce the complexity of the control law it is assumed that the Kalman filter above, specifies $p\left(x_{k} \mid I_{k-1}\right)$ exactly, i.e. constitutes a sufficient statistics, [7]. Hence

$$
p\left(x_{k} \mid I_{k-1}\right)=N\left(\hat{x}_{k \mid k-1}, \Sigma_{k \mid k-1}\right)
$$

where $\hat{x}_{k \mid k-1}$ and $\Sigma_{k \mid k-1}$ are given by (15) and (16).

The minimization problem (3) is considered for the dynamics (10) and observation model (13) and the control law at time $t_{k}$ should be a function of (19). In order to study basic principles we simplify by putting $M_{k}=0$ and $S_{k}=0$ for $k=0,1, \ldots, N-1$. Using dynamic programming (3) is to be solved backwards starting with $u_{N-1}$. At time $t_{N-1}$ (3) is given as

$E\left[\left(x_{N}-x_{g}\right)^{2}\right]=\underset{I_{N-2}}{E} E\left[\left(x_{N}-x_{g}\right)^{2} \mid I_{N-2}\right]$

when $S_{N}=1$.

It is well known by lemma (3.2) in [3] that for a unique minimum $u_{N-1}^{*}$ the outer expectation above do not affect the minimization. Hence the cost to minimize at time $t_{N-1}$, denoted $\bar{J}_{N-1}$, is

$$
\begin{aligned}
& \bar{J}_{N-1}\left(\hat{x}_{N-1 \mid N-2}, \Sigma_{N-1 \mid N-2}\right)=\min _{u_{N-1}} E\left[\left(x_{N-1}+T u_{N-1}+\right.\right. \\
& \left.\left.b u_{N-1} \sqrt{T} \tilde{w} 1_{N-1}+c \sqrt{T} \tilde{w} 2_{N-1}-x_{g}\right)\left.^{2}\right|_{I_{N-2}}\right]
\end{aligned}
$$

where $x_{N}$ is expressed using the motion model (10). After taking expectation $\bar{J}_{N-1}$ is given as

$$
\begin{aligned}
& \min _{u_{N-1}}\left[\left(\hat{x}_{N-1 \mid N-2}+u_{N-1} T-x_{g}\right)^{2}+\Sigma_{N-1 N-2}+\right. \\
& \left.b^{2} u^{2}{ }_{N-1} T+c^{2} T \mid I_{N-2}\right]
\end{aligned}
$$

The cost is quadratic in $u_{N-1}$ and the minimizing control signal for the last step $u_{N-1}^{*}$ is given as

$$
u_{N-1}^{*}=\frac{x_{g}-\hat{x}_{N-11 N-2}}{T+b^{2}}
$$

The resulting cost-to-go function $\bar{J}_{N-1}$ is given as

$$
\begin{aligned}
& \bar{J}_{N-1}\left(\hat{x}_{N-1 \mid N-2}, \Sigma_{N-1 \mid N-2}\right)=\Sigma_{N-1 \mid N-2}+ \\
& \frac{b^{2}\left(x_{g}-\hat{x}_{N-1 \mid N-2}\right)^{2}}{T+b^{2}}+c^{2} T
\end{aligned}
$$

Note that when using (22) in the motion model (10) with $b=0$, the controller removes the expected value of the control error completely, i.e. a deadbeat property. $\bar{J}_{N-1}$ can be physically interpreted as the minimal value of $\Sigma_{N \mid N-2}+\left(\hat{x}_{N \mid N-2}-x_{g}\right)^{2}$. Clearly the last step $u_{N-1}$ cannot 
benefit from any probing, i.e. control actions in purpose to only reduce estimation uncertainty.

\subsection{Two Steps-To-Go}

At time $t_{N-2}, u_{N-2}$ is to be derived. The cost to minimize is then given by the expected value of $\bar{J}_{N-1}$, hence

$$
\begin{aligned}
& \bar{J}_{N-2}\left(\hat{x}_{N-2 \mid N-3}, \Sigma_{N-2 \mid N-3}\right)=\min _{u_{N-2}} \\
& E\left[\bar{J}_{N-1}\left(\hat{x}_{N-1 \mid N-2}, \Sigma_{N-1 \mid N-2}\right) \mid I_{N-3}\right]=\min _{U_{N-2}} E \\
& {\left[\Sigma_{N-1 \mid N-2}+\frac{b^{2}\left(x_{g}-\hat{x}_{N-1 \mid N-2}\right)^{2}}{T+b^{2}}+c^{2} T \mid I_{N-3}\right]}
\end{aligned}
$$

where $\hat{x}_{N-1 W-2}$ and $\sum_{N-1 N-2}$ are given by (15) and (16). Clearly both $\hat{x}_{N-1 \mid N-2}$ and $\sum_{N-1 N-2}$ are random variables since they include $z_{N-2}$ and $\gamma_{N-2}$. Further, (24) is written as

$$
\begin{aligned}
& \min _{u_{N-2}} \underset{\gamma_{N-2}}{E} \underset{z_{N-2}}{E}\left[\Sigma_{N-1 \mid N-2}+\right. \\
& \left.\frac{b^{2}\left(x_{g}-\hat{x}_{N-1 \mid N-2}\right)^{2}}{T+b^{2}}+c^{2} T \gamma_{N-2}, I_{N-3}\right]
\end{aligned}
$$

Now the inner expectation is evaluated assuming (13) and (19) apply, i.e. that $z_{k}$ is a normal variable, hence

$$
\begin{aligned}
& \min _{u_{N-2}} \gamma_{N-2}^{E}\left[\Sigma_{N-2 \mid N-3}\left(1-\gamma_{N-2}\right)+b^{2} u_{N-2}^{2} T+\right. \\
& \frac{R_{N-2} \Sigma_{N-2 \mid N-3}}{H_{N-2}^{2} \Sigma_{N-2 \mid N-3}+R_{N-2}} \gamma_{N-2}+2 c^{2} T+ \\
& \frac{b^{2}}{T+b^{2}}\left(\left(x_{g}-\hat{x}_{N-1 \mid N-2}-u_{N-2} T\right)^{2}+\right. \\
& \left.\left.\frac{H_{N-2}^{2} \Sigma_{N-2 \mid N-3}^{2}}{H_{N-2}^{2} \Sigma_{N-2 \mid N-3}+R_{N-2}} \gamma_{N-2}^{2}\right)\left.\right|_{I_{N-3}}\right]
\end{aligned}
$$

The other expectation in (26) over $\gamma_{N-2}$ is evaluated by using the probability of receiving a measurement $z_{N-2}$. Let $P_{k}$ (1) be the probability that $z_{k}>z_{\max }$, Figure 2 .

$$
\begin{aligned}
& P_{k}(1)=\operatorname{Prob}\left(z_{k}>z_{\max } \mid I_{k-1}\right)= \\
& 1-\Phi\left(\frac{z_{\max }-h\left(\hat{x}_{k \mid k-1}\right)}{\sqrt{H_{k}^{2} \Sigma_{k \mid k-1}+R_{k}}}\right)
\end{aligned}
$$

where $\Phi$ is the distribution of a $\mathrm{N}(0,1)$ variable. In the same way we have $P_{k}$ (2) for $z_{k}<z_{\text {min }}$. Let further $P_{k}$ denote the total probability that $z_{k}$ is outside the field of view, i.e. $P_{k}=P_{k}(1)+P_{k}(2)$. Now the outer expectation in (26) is evaluated giving

$$
\begin{aligned}
& \min _{u_{N-2}}\left[\Sigma_{N-2 \mid N-3} P_{N-2}+b^{2} u_{N-2}^{2} T+2 c^{2} T+\right. \\
& \frac{R_{N-2} \sum_{N-2 \mid N-3}}{H_{N-2}^{2} \sum_{N-2 \mid N-3}+R_{N-2}}\left(1-P_{N-2}\right)+ \\
& \frac{b^{2}}{T+b^{2}}\left(\left(x_{g}-\hat{x}_{N-1 \mid N-2}-u_{N-2} T\right)^{2}+\right. \\
& \left.\left.\frac{H_{N-2}^{2} \sum_{N-2 \mid N-3}^{2}}{H_{N-2}^{2} \sum_{N-2 \mid N-3}+R_{N-2}}\left(1-P_{N-2}\right)\right)\right]
\end{aligned}
$$

Clearly (28) is also quadratic in the control function and can be minimized analytically, hence

$$
u_{N-2}^{*}=\frac{x_{g}-\hat{x}_{N-2 \mid N-3}}{2 T+b^{2}}
$$

The corresponding cost-to-go $\bar{J}_{N-2}$ is given by

$$
\begin{aligned}
& \bar{J}_{N-2}=\Sigma_{N-2 \mid N-3} P_{N-2}+2 c^{2} T+\frac{b^{2}\left(x_{g}-\hat{x}_{N-2 \mid N-3}\right)^{2}}{\left(2 T+b^{2}\right)}+ \\
& \left(1-P_{N-2}\right)\left(\frac{R_{N-2} \Sigma_{N-2 \mid N-3}}{H_{N-2}^{2} \sum_{N-2 \mid N-3}+R_{N-2}}+\right. \\
& \left.\frac{b^{2} H_{N-2}^{2} \sum_{N-2 \mid N-3}^{2}}{\left(T+b^{2}\right)\left(H_{N-2}^{2} \sum_{N-2 \mid N-3}+R_{N-2}\right)}\right)
\end{aligned}
$$

The cost-to-go $\bar{J}_{N-2}$, Figure 4 , can also be given a physical interpretation. In order to describes the minimal value of the expected final uncertainty plus expected final quadratic position error we get the new last term. It is interpreted as a cost of including the measurement $z_{N-2}$. Not even at time $t_{N-2}$ can the control law benefit from probing. A probing term in $u_{N-2}$ would not give a reduction in estimation uncertainty before the final time is reached.

\subsection{The N-Step Control Law}

In order to derive the remaining control steps $\left(u_{0}, \ldots u_{N-3}\right)$ we proceed recursively starting at time $t_{k}=t_{N-3}$, hence

$$
\bar{J}_{k}\left(p\left(x_{k} \mid I_{k-1}\right)\right)=\min _{u_{k}} E\left[\bar{J}_{k+1}\left(p\left(x_{k+1} \mid Z^{k}\right)\right) \mid I_{k-1}\right]
$$

with the minimizing control law of the form $u_{k}^{*}\left(p\left(x_{k} \mid I_{k-1}\right)\right)=u_{k}^{*}\left(\hat{x}_{k+1 k}, \Sigma_{k+1 k}\right) k=0,1, ., N-1$

Before minimizing (30) w.r.t. $u_{N-3}$ we must calculate the expectation. By examining (30) using the filter equations (15) (16) and (14), we conclude that the cost is a nonlinear function w.r.t. the random variables $z_{N-3}$ and $\gamma_{N-3}$. By introducing $g$ such that $\vec{J}_{N-3}=g\left(z_{N-3}, \gamma_{N-3}, I_{N-4}\right)$ we write the expectation as

$$
\begin{aligned}
& E\left[\left.g\left(z_{N-3}, \gamma_{N-3}, I_{N-4}\right)\right|_{N-4}\right]= \\
& \gamma_{N-3}^{E} \underset{z_{N-3}}{E}\left[g\left(z_{N-3}, \gamma_{N-3}, I_{N-4}\right) \mid \gamma_{N-3}, I_{N-4}\right]= \\
& P_{N-3} g\left(z_{N-3}, 0, I_{N-4}\right)+\left(1-P_{N-3}\right) \\
& \int_{-\infty}^{+\infty} g\left(z_{N-3}, 1, I_{N-4}\right) p\left(z_{N-3} \mid I_{N-4}\right) d z_{N-3}
\end{aligned}
$$


where $P_{N-3}$ is the total probability that $z_{N-3}$ is outside the field of view, $\mathrm{z} \in\left[\mathrm{z}_{\min }, \mathrm{z}_{\max }\right]$. Also, the cost $\bar{J}_{N-2}$ is highly non-linear w.r.t. $u_{N-3}$ with several local minima. These two complications makes it impossible to proceed analytically without further approximations. Consequently the variables $\hat{x}_{N-3 \mid N-4}$ and $\Sigma_{N-3 \mid N-4}$ are discretized and a minimizing $u_{N-3}$ is found for every grid point in the $\left(\hat{x}_{N-3 \mid N-4}, \Sigma_{N-3 \mid N-4}\right)$ space. The maximal value for $\Sigma_{N-3 \mid N-4}$ is chosen large enough to make it possible to study consequences of linearization errors during simulation, Figure 7 and 8 . The integral in (32) is solved by a quadrature formula and the numerical minimization is made using a gradient method starting in the vicinity of the global minima for every grid point. In Figure 5,6 and 7 the result of solving (31) numerically for $N=3, \ldots, 5$ are presented. The calculation procedure for $N=4$ and 5 follows the one described for $N=3$.

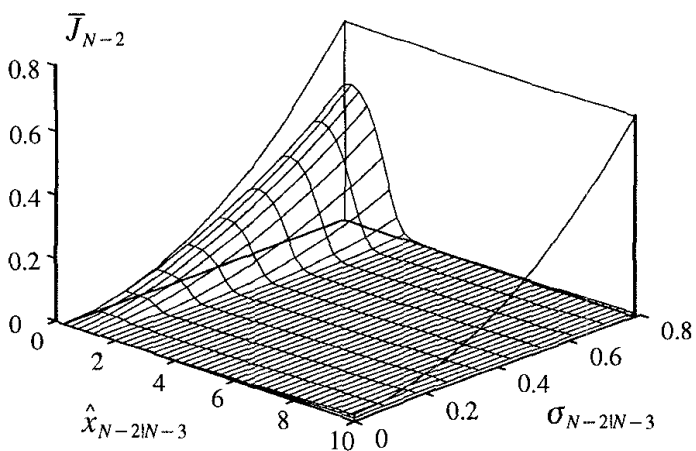

Figure 4. Mesh plot of the two-step cost-to-go function $\bar{J}_{N-2}$ with respect to the position estimate and corresponding standard deviation. Note the increase in cost for start positions inside the blind zone. Also the shape of one step $\bar{J}_{N-1}$ is outlined.

\section{THE FAILURE PROBABILITIES}

In this section we will calculate an approximate probability for a failure when positioning the robot. A failure means that the robot is not positioned within the given tolerances at the last step. The probability is a prediction before the actual control law is applied. Such a probability can tell us much about the forthcoming task. First, for a given control law and failure probability $p_{f}$ we get an upper bound on the initial uncertainty $\Sigma_{0}$. Also given $p_{f}, \epsilon$ and $\Sigma_{0}$ one can estimate how many of the planned control steps that need to be used. For instance $\Sigma_{0}$ might be so low that the last $\mathrm{m}$ steps of an $\mathrm{N}$-step control law are sufficient. In the original formulation of the problem we want to calculate if the probability of failure is acceptable, i.e.

$\operatorname{Prob}\left(\left(x_{N}-x_{g}\right) \notin A\right)<p_{f}$
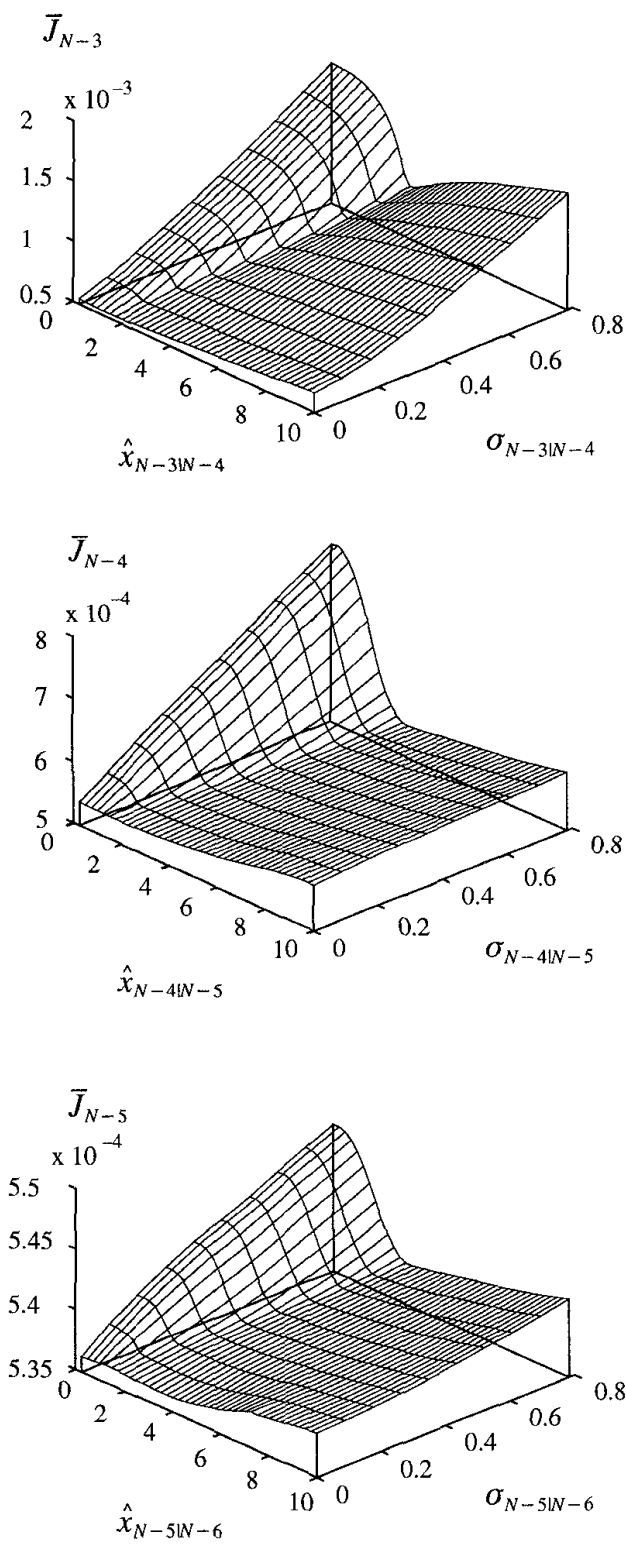

Figure 5. The cost-to-go for the control law with $N=3,4$ and 5 respectively. The increasing cost for constant standard deviation and increasing range is due to the range dependent noise. Note also how the relative importance of the start position is decreasing for longer control horizon. The small hollow at $x=4$ in $J_{N-5}$ is due to numerical errors during minimization.

First introduce the functional describtion giving the position estimate and variance, i.e. the estimator, hence 


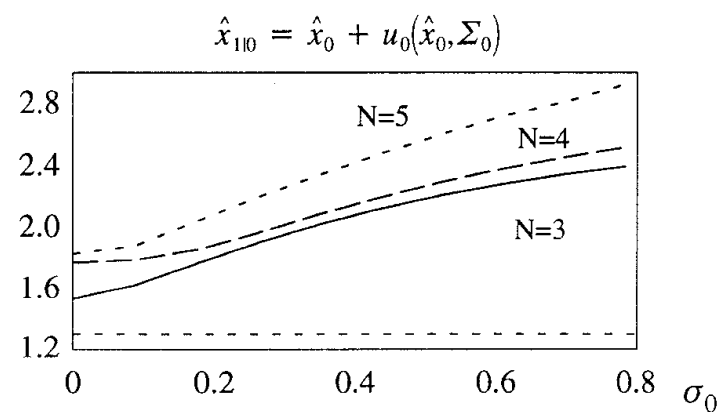

Figure 6. The predicted position after the first control step with respect to the initial uncertainty. The horizontal line marks the blind zone for the sensor. The 3 curves are derived with different control horizon, i.e. $N=3,4$ and 5 . Note the cautious behaviour for all 3 w.r.t. the blind zone for increasing initial uncertainty. Alltough the best measurement position is just in front of the blind zone the control law with longer control horizon plans for not to large initial control steps in order to keep the motion error low.

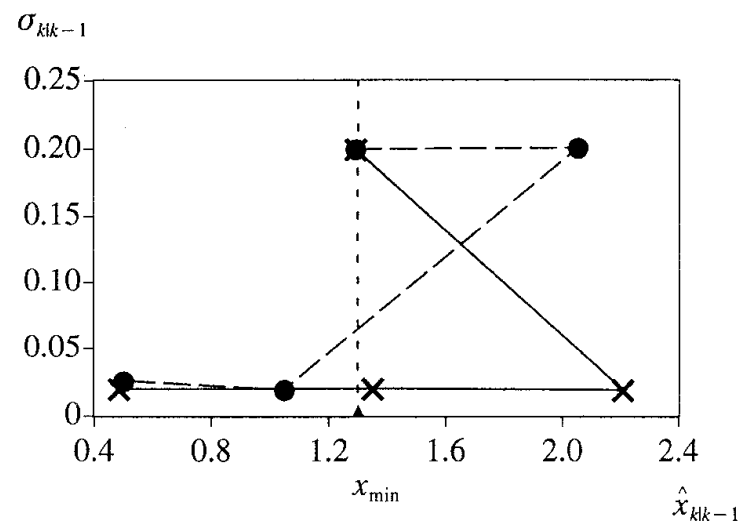

Figure 7. Two typical trajectories in $\left(x_{\left.k \mid k-1, \sigma_{k \mid k-1}\right)}\right.$ space. The control law only plan one measurement, i.e. $N=3$. The vertical dotted line marks the blind zone. The initial position was chosen randomly $N\left(x_{\min }, 0.2\right)$. From the plot it is clear that one of the simulations do not get an initial measurement. However the other simulation even gets a measurement at $t_{N-1}$. Using 1000 simulations the standard deviation of the true final position was $\approx 0.03$. This is consequently below the limit in initial uncertainty were the disagreement with the variance in the Kalman filter starts.

$$
\begin{aligned}
X^{*}{ }_{k+1 k k} & =E\left[x_{k+1} \mid\left(Z_{0}, \ldots, Z_{k}, U_{0}, \ldots, U_{k}\right)\right] \\
\Sigma^{*}{ }_{k+1 \mid k} & =E\left[\left(x_{k+1}-X^{*}{ }_{k+11 k}\right)^{2} \mid\left(Z_{0}, \ldots, Z_{k}, U_{0}, \ldots U_{k}\right)\right]
\end{aligned}
$$

The estimator is given by the same equations as the estimate, i.e. (15) and (16), with the only difference that the observation $z_{k}$ is interchanged with $Z_{k}$, i.e. a random observa- tion and $U_{k}=u_{k}\left(X_{k \mid k-1}^{*}, \Sigma_{k \mid k-1}^{*}\right)$. Denote the expected value of (34) by $\hat{X}_{k+1 \mid k}^{*}$ and $\hat{\Sigma}^{*}{ }_{k+1 \mid k}$. Then we want to derive $\hat{X}_{N N-2}^{*}$ and $\hat{\Sigma}_{N N-2}^{*}$. Note here that we are only interested in the mean and variance of the estimator conditioned on the random observations up to $t_{N-2}$. This since the observation at $t_{N-1}$ is not available before the final time $t_{N}$. The above expected values are however non-linear in the random observations and in the random variables modelling the motion noise. A first order approximation of them are given by replacing all random variables by there mean value. The expectations can then be calculated iteratively from the following recursive scheme

$$
\begin{aligned}
& \hat{X}_{k+1 \mid k}^{*}=\hat{X}_{k \mid k-1}^{*}+T u_{k}\left(\hat{X}_{k k-1}^{*}, \hat{\Sigma}_{k \mid k-1}^{*}\right) \\
& \hat{\Sigma}_{k+1 \mid k}^{*}=\hat{\Sigma}_{k \mid k-1}^{*}\left(1-\gamma_{k}^{*}\right)+\frac{\hat{\Sigma}_{k \mid k-1}^{*} R}{H^{*^{2}}{ }_{\Sigma^{*}}^{*}{ }_{k \mid k-1}+R} \gamma_{k}^{*} \\
& +b^{*} u_{k}\left(\hat{X}_{k \mid k-1}^{*}, \Sigma_{k \mid k-1}^{*}\right)^{2} T+c^{2} T
\end{aligned}
$$

with start values given by the prior $p\left(x_{0}\right)$, i.e. ${\hat{X^{*}}}_{0}=x_{0}$ and $\hat{\Sigma}^{*}{ }_{0}=\Sigma_{0}$. The value of $H^{*}{ }_{k}$ and $\gamma_{k}{ }_{k}$ in (35) are given by using $\hat{X}_{k \mid k-1}^{*}$ and $h\left(\hat{X}_{k \mid k-1}^{*}\right)$ instead of $\hat{x}_{k \mid k-1}$ and $z_{k}$ in (14) and (7) respectively. The sought expectations are then given by $X_{N N-1}^{*}=X_{N N-2}^{*}$ and $\Sigma^{*}{ }_{N-11 N-2}+b^{2} u^{2}{ }_{N-1}\left(\hat{X}^{*}{ }_{N-1}\right) T+c^{2} T$. However the expected final variance, i.e. $\hat{\Sigma}_{N N-2}^{*}$ is also given, without the approximations, from the cost-to-go $\bar{J}_{0}$, since

$$
E\left[\Sigma_{N I N-2}^{*}\right]=\bar{J}_{0}-\left(\hat{X}_{N N-2}^{*}-x_{g}\right)^{2}
$$

Using (36) and $\hat{X}_{N I N-2}^{*}$ we now have an approximation of the p.d.f. of the final state $x_{N}$. In Figure 8 , $\operatorname{Prob}\left(\left(x_{N}-x_{g}\right) \notin A<p_{f}\right)$ is plotted versus $\frac{\sigma_{0}}{\epsilon}$ for fixed tolerance $\epsilon$ for the control law with a 3,4 and 5 step horizon. $\sigma_{0}$ is the square root of the initial uncertainty $\Sigma_{0}$. The set $A$ is given by the distance $\pm \epsilon$ around $x_{g}$. The failure probability is compared with a Monte Carlo simulation of the system given by (10) and (7) using the feedback controller. The graphs readily limits the initial uncertainty $\Sigma_{0}$ for given tolerances and failure probability.

\section{CONCLUSIONS}

The problem addressed was to design a feedback controller when a range camera is used for positioning of a robot relative an object within prespecified tolerances. For a 1 -degree-of-freedom robot an approximative solution was derived using dynamic programming. A 5-step control law is evaluated. Typically, during the first 3 steps the con- 

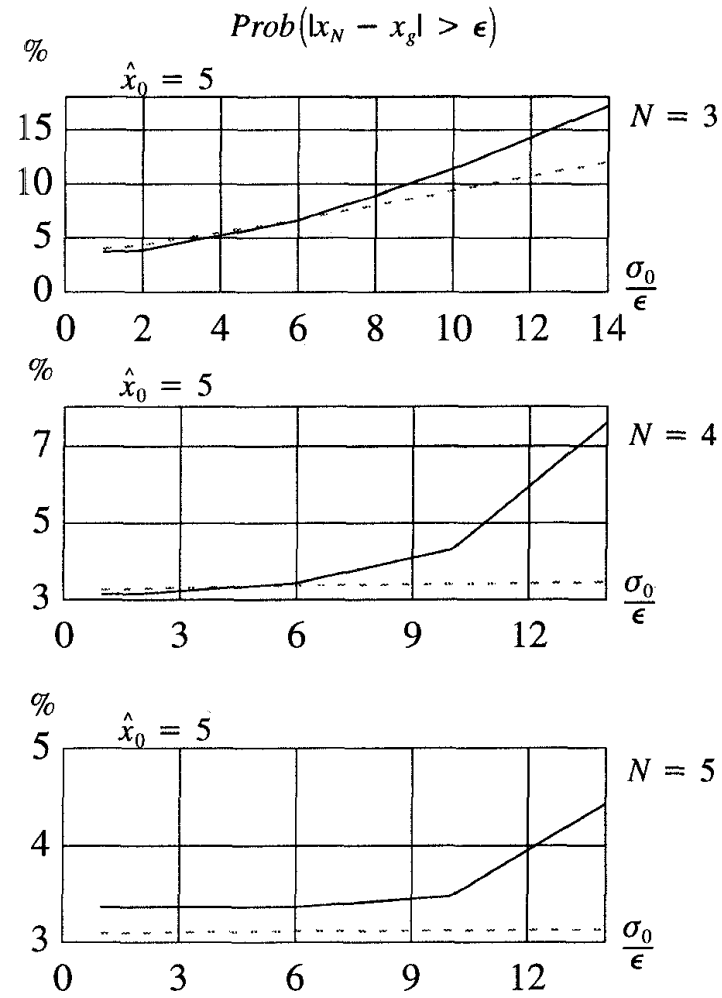

Figure 8. The predicted failure \% (dotted line) compared to a Monte Carlo simulation for $N=3,4$ and 5 . The prediction is within $\pm 2 \%$ of the true failure rate up to a limiting start uncertainty. For larger start uncertainty the linear approximation in the measurement equation (13) are not valid. $b^{2} T=c^{2} T=2.3 E-4$ was used wich corresponds to a standard deviation for the noise term giving constant motion uncertainty of $\sim 0.6[\mathrm{~mm}]$ when $h=4[\mathrm{~cm}]$. and $R=2 E-7$ corresponds to a lab sensor.

trol law positions the robot at the expected best measurement position, i.e. probing. Subsequently the control law steers the robot to the correct gripping point. The control law is also capable of reducing the uncertainty when the object is outside the camera field of view. It actively, not accidently, steers the robot out of the blind zone. This behavior is not automatic with an ordinary certainty-equivalence controller.

The dynamic programming framework used in the design is generic in handling any uncertainty, however suffers from increasing complexity for higher order systems.

Future work will be focused on a corresponding controller for a 3-degree-of-freedom SCARA robot. Then decoupling of the control law must be considered. An algorithm for the necessary number of control steps with respect to initial uncertainty and system parameters is also needed.
In the future it might be possible to control robots using kinematic, dynamic and sensor models. The task coordinates and tolerances are specified by a planner and the feedback laws are synthesized by optimization. This paper might be one small contribution towards the necessary theoretical basis for such systems.

\section{References}

[1] Bertsekas, D.P., Dynamic Programming and Stochastic Control. Academic Press, 1976.

[2] Hager, G.D., Task-Directed Sensor Fusion and Planning. Kluwer Academic Publishers, Boston, 1990.

[3] Åström, K.J., Introduction to Stochastic Control Theory. Academic Press, 1970.

[4] Wernersson, Å., Boberg, B., Nilsson, B., Nygårds, J., and Rydberg, T., On sensor feedback for gripping an object within prescribed posture tolerances. Proc. IEEE Int. Conf. on Robotics and Automation, Nice, 1992, pp. 2012-2017.

[5] Nygårds, J., Wernersson, A., Specular and Transparent Objects in Moving Range Cameras: Active Reduction of Ambiguities, Preprint, SPIE vol. 2534, Boston 94.

[6] Hashimoto, K., Visual Servoing:Real-Time Control of Robot Manipulators Based on Visual Sensory Feedback. World Scientific Publishing Co., 1993.

[7] Aoki, M.,Optimization of Stochastic Systems. Academic Press , 1967.

[8] El-Hakim, S.F., Beraldin J.-A., On the Integration of Range and Intensity Data to Improve VisionBased Three-Dimensional Measurements, Proc. SPIE vol. 2350, Boston 1994.

[9] Cannata, G., Grosso E., Active Eye-Head Control, Proc. IEEE Int. Conf. on Robotics and Automation, San Diego, 1994, pp. 2837-2843.

[10] Roth, G., O'Hara, D., Levine, M.D., A Holdsite Method for Parts Acquisition Using a Laser Rangefinder Mounted on a Robot Wrist, Journal of Robotic Systems, 6(5), pp. 573-599 (1989).

[11] Bajcsy, R., Active perception, Proc. IEEE special issue on Computer Vision, pp. 996-1005, August 1988.

[12] Zheng, J.Y., Chen, Q., Tsuji, S., Active Camera Guided Manipulation, Proc. IEEE Int. Conf. on Robotics and Automation, Sacramento, California, 1991, pp. 632-638. 